\title{
Hippocampal differentially expressed genes between tame and aggressive foxes are included in pathways associated with stress, behavior and adult neurogenesis
}

Yu. Herbeck*, M. Genaev, G. Vasiliev, R. Gulevich, S. Shikhevich, D. Shepeleva, D. Afonnikov, L. Trut Institute of Cytology and Genetics SB RAS, Novosibirsk, Russia

*e-mail: herbek@bionet.nsc.ru

Key words: domestication, stress, NGS

Motivation and Aim: The experimental domestication of foxes has demonstrated that essential mechanism of domestication is the decrease of stress response in particular toward human. Previously it was shown reduced plasma basal and stress-induced cortisol levels in tame foxes [1]. However, the molecular genetic mechanisms of these changes remain unclear. The aim of this work is to identify differentially expressed genes (DEG) in the hippocampus of domesticated ('tame') and aggressive foxes. The hippocampus is the key site of glucocorticoid negative feedback [2].

Methods and Algorithms: Single-read 75 bp sequencing of hippocampal cDNA libraries was performed using Illumina HiSeq ( $n=3$ foxes per behavior group, on average $40 \mathrm{mln}$ reads per sample). The DEG analysis was performed by the Cufflink (cole-trapnell-lab. github.io/cufflinks). The dog genome was used as a reference, and Ensemble rev. 86 was used as an annotation. Over-representation analysis was performed using web-based gene set analysis toolkit (WebGestalt) (www.webgestalt.org).

Results: Analysis of 496 DEGs detected in the hippocampus of tame and aggressive foxes revealed only 10 pathways significantly associated with DEG. Most of these pathways are associated with the nervous system, behavior, aggression, stress and neurogenesis. Conclusion: Apparently, the differences in gene expression in the hippocampus between tame and aggressive foxes are primarily associated with signal transmission (cAMP and calcium signaling pathways), neurogenesis and axon guidance, adrenergic signaling, opioid system (amphetamine addiction). These pathways and individual DEGs play an important role in stress reactivity, behavior and adult neurogenesis.

Acknowledgements: Supported by the RSF (No. 16-14-10216).

\section{References}

1. Trut L.N. et al. (2009) Animal evolution during domestication: the domesticated fox as a model. BioEssays. 31(3):349-360.

2. Jacobson L., Sapolsky R.M. (1991) The role of the hippocampus in feedback regulation of the hypothalamo-pituitary-adrenocortical axis. Endocr. Rev. 12:118-134. 